\title{
An installation for transport and accumulation studies on undisturbed soil columns under controlled conditions
}

\author{
D. W. Bannink, P. Poelstra ${ }^{1}$ and M. J. Frissel
}

Institute for Atomic Sciences in Agriculture (ITAL), P.O. Box 48, Wageningen, the Netherlands

Accepted: 31 March

Key words:

\section{Summary}

An installation is described which enables transport and accumulation studies to be carried out on undisturbed soil columns under controlled conditions. An adjustable constant water flux can be maintained and the influent is applied as drops to the top of the soil column. Soils are not saturated with water. Aerobic as well as anaerobic conditions can be maintained during the experiment. A technique is described to take undisturbed soil columns from the field to a depth of $1 \mathrm{~m}$. It is easy to apply, cheap and reliable.

\section{Introduction}

Transport and accumulation studies in soils have been intensified in the past years, due to the need for information on the risks of soil pollution. For this reason an installation has been developed which enables transport and accumulation studies to be carried out on undisturbed soil columns under controlled conditions. The installation meets the following requirements.

1. The columns are taken from the field in such a way that the natural profile is maintained.

2. It is provided with a rain simulator to supply the influent as drops.

3. Visual inspection of the system is possible by using transparent material for columns and rain simulators.

4. The columns are not water-saturated; it is possible to simulate each reasonable phreatic level. When water saturation is required, the system can be adapted to it. 5. Aerobic as well as anaerobic conditions can be maintained (without water saturation).

6. Tensiometer gauges and electrodes for water potential, $\mathrm{pH}$ and redox potential measurements can be inserted into the column.

1 All correspondence should be directed to P. Poelstra. 
7. The soil core can be cut into slices at the end of an experiment without damaging the cylinder.

8. The soil columns can easily be scanned when the compound under investigation is labelled with a $\gamma$-emitting isotope.

9. The soil columns can easily be scanned by a 'double-beam' scanner to determine moisture and soil density.

A description of the system together with the techniques developed to take the columns from the field is presented below.

\section{Preparation of the soil columns}

The material used for the soil columns is perspex. The cylinders each have a $6 \mathrm{~mm}$ thick wall, an internal diameter of $120 \mathrm{~mm}$ and a length of $1 \mathrm{~m}$. They are all provided with a screw-thread. A hollow auger-head of hardened steel is screwed onto the cylinder. Two anchors with a length of $2 \mathrm{~m}$ (looking like a corkscrew) are turned into the soil (see Fig. 1). A bar is pushed through rings fixed on top of each anchor, a block of wood is placed on top of the cylinder and an oil-jack set between block and bar. The cylinder is gradually pressed into the soil. When distance between bar and block can no longer be overlapped by the jack, another block is used. The jack is then placed on top of a second cylinder and, after pressing, on a third one. The three of them are pressed into the soil to a certain depth, then the anchors are turned deeper into the soil (with the bar) and the pressing process is continued. Finally the columns are dug out. If more than three soil columns are needed a second row of three is made in front of the first row to save digging work. The jack is provided with a pressure meter to observe resistance. Stones are also detected in this way and may be dug out. If oil pressure reaches more than 90 to 100 atmospheres ( $\sim 9$ to $10 \mathrm{MPa}$ ), soil around the cylinder must be partly removed.

The internal diameter of the auger head is 1 to $2 \mathrm{~mm}$ less than the internal diameter of the cylinder. Experience has shown that compression of the soil core

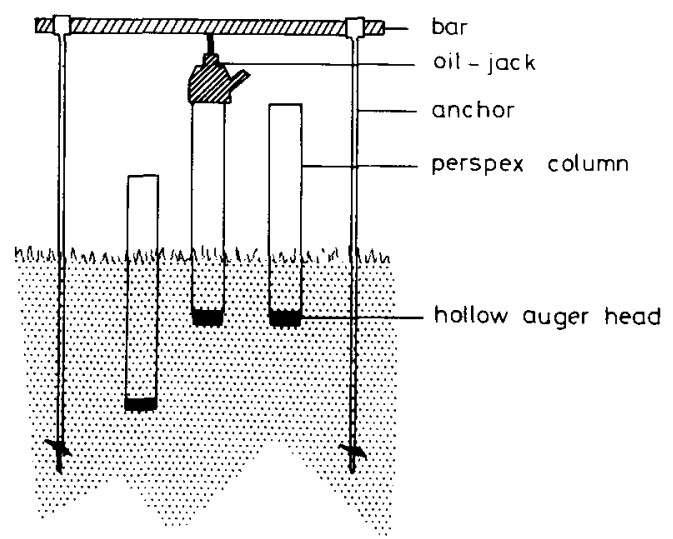

Fig. 1. Scheme of a manual method to take undisturbed soil columns in the field. The columns have a length of 1 metre and an internal diameter of $12 \mathrm{~cm}$. 
is less than $1 \%$ and no risk of cylinder cracking exists. The small space between soil core and cylinder wall does not influence the leaching pattern.

This manual method may be modified to a mechanical method as demonstrated at the Laboratory for Soils and Plant Nutrition of the Nuclear Research Centre 'Democritos' in Athens during a visit of M. J. Frissel.

A stainless steel cylinder fitted on an available motorized auger was provided with a dismountable auger-head. The perspex cylinder described above just fits into this steel cylinder. This mechanical method will be described elsewhere (E. P. Papanicolaou \& C. G. Apostolakis, pers. comm.).

If the phreatic level is considerably higher than $1 \mathrm{~m}$, both methods can still be applied, provided well-pumping is done. The phreatic level is then lowered locally.

\section{Description of the soil column installation}

An overall picture of the soil column installation is shown in Fig. 2. The soil columns are fixed in racks, provided with a filter underneath and connected to an automized system for supplying the leaching solution as drops with an adjustable constant flux.

A scheme of this system is shown in Fig. 3. A time-switch operates a magnet valve. When it is opened the pneumatic system presses an adjustable volume of

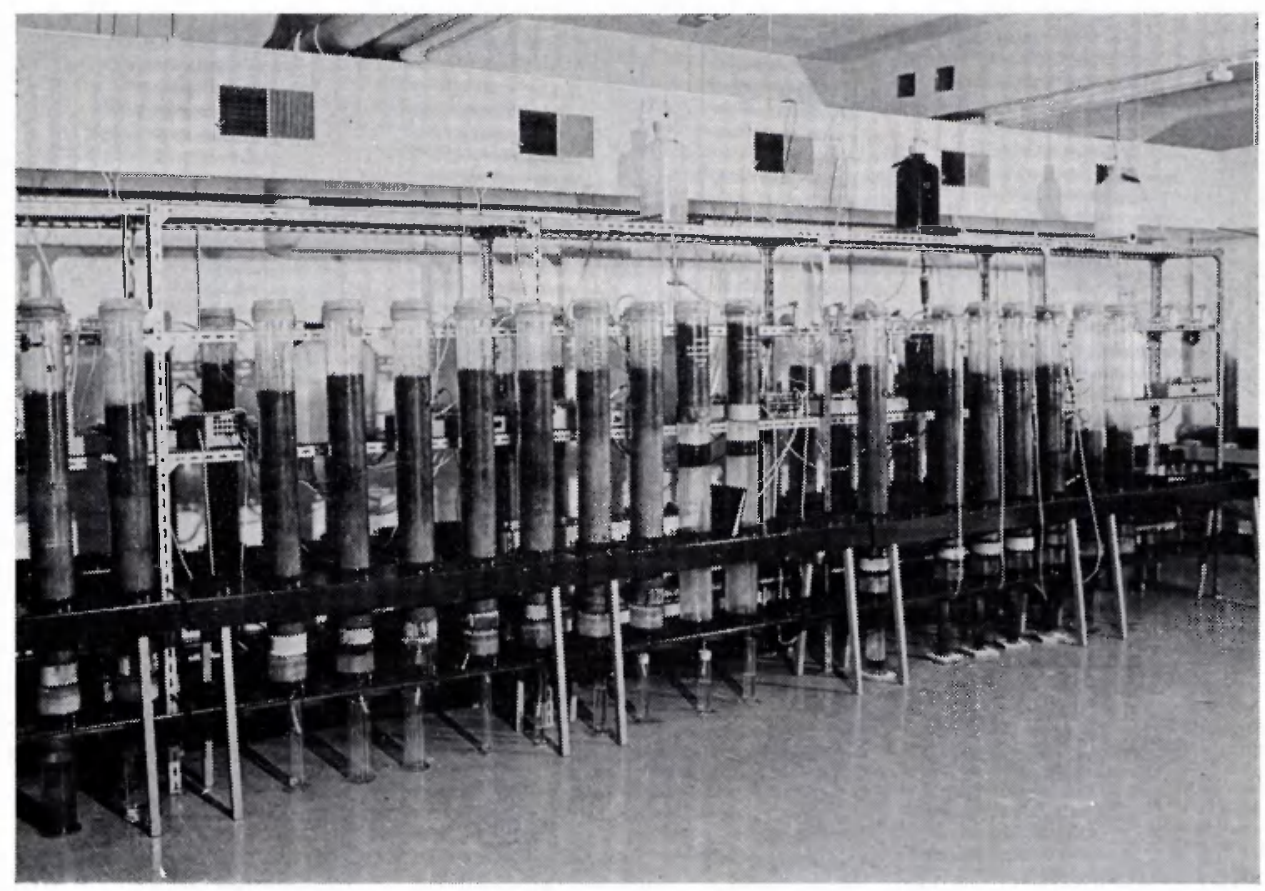

Fig. 2. Overall picture of the soil column installation. 


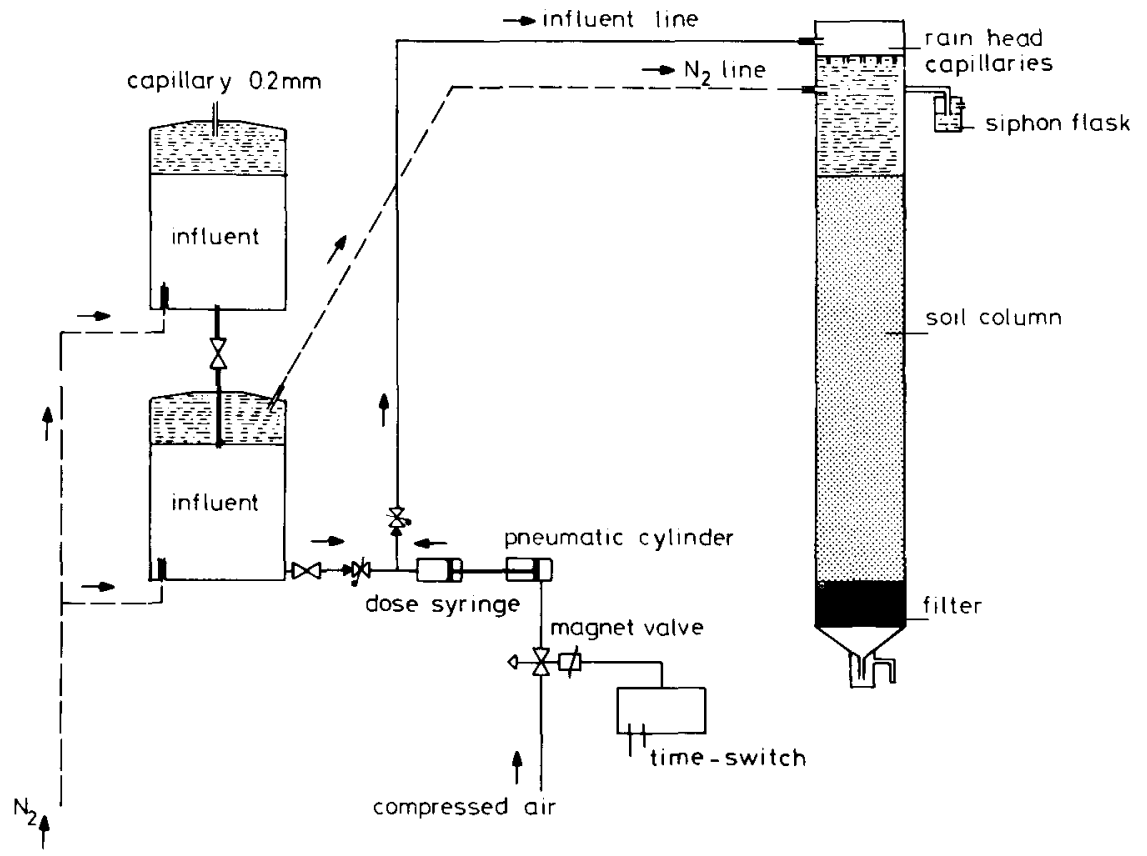

Fig. 3. Scheme of the soil column installation. For explication see text.

influent into the rain-head placed on top of each column. Consistent with the construction of the rain-head the same volume then leaves the rain-head as drops via 25 capillaries fixed in the lower plate. When the magnet valve is closed a spring construction in syringe and cylinder re-sets both to their original positions. The syringe is adjustable from 1 to $5 \mathrm{ml}$, the time-switch from 5 to 60 minutes. Adjustment of both supplies the required flux.

\section{The rain-head}

The rain-head is made of the same material as the cylinder for the soil column. It is also a cylinder, of the same diameter, but with a height of $5 \mathrm{~cm}$. Twenty-five capillaries each with a length of $35 \mathrm{~mm}$ are fixed in the lower plate, according to such a distribution pattern that each capillary covers an equal area. The internal diameter of the capillary may range from 0.2 to $1.0 \mathrm{~mm}$. Experience has shown that beyond $0.5 \mathrm{~mm}$ formation of drops no longer occurs; the solution then leaves the rain-head as a tiny stream. The choice of the internal diameter of the capillaries is based on the kind of influent to be used. If it contains small insoluble particles, larger diameters should be used to avoid blocking of the capillaries. Some blocking does not influence the quantity of influent delivered to the soil column. Only the regular distribution over the soil surface is then disturbed. The rain-head should be made air-tight; if not, it empties itself through the capillaries and makes the flux irregular. 


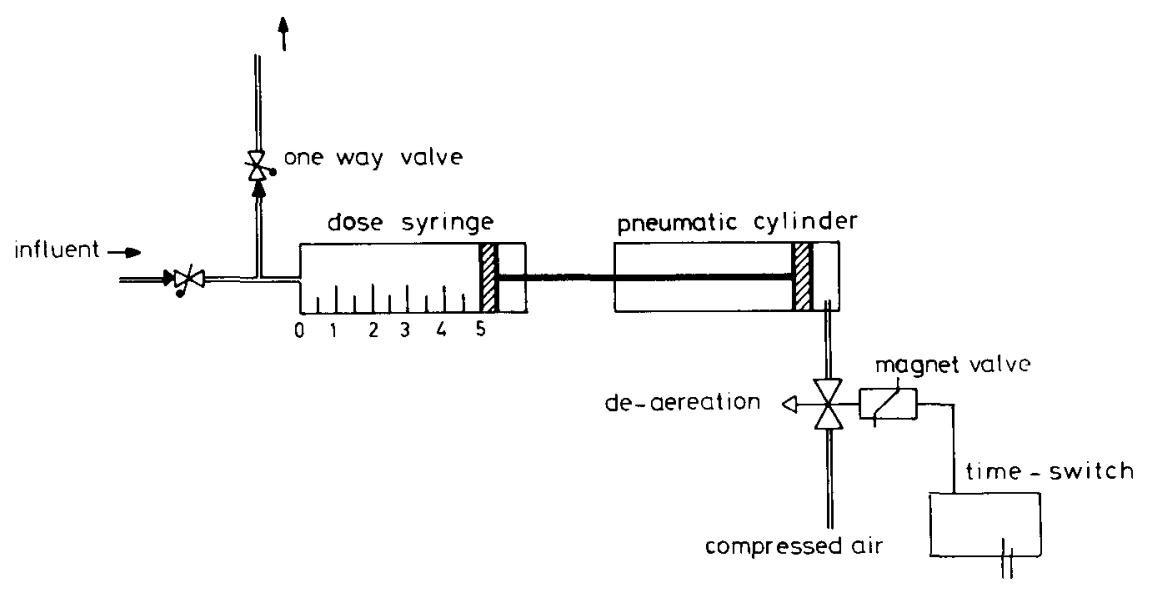

Fig. 4. Detail of the scheme of the soil column installation.

The capillaries are fixed in the lower plate by a simple technique. The plate is perforated with 25 holes, each having a diameter $0.1 \mathrm{~mm}$ smaller than the outside diameter of the capillaries. The perforated plate is then placed on a hot plate kept at $80{ }^{\circ} \mathrm{C}$ for some time. It then weakens and the capillaries can be pressed into the holes with a plastic hammer. After cooling they are tightly fixed in the plate. For safety reasons they are also glued.

The pneumatic system

Each rain-head has its own pneumatic system. This means that the flux can be adjusted independently for each rain-head. The pumping part consists of an adjustable dose syringe, the mechanical part of a compressed air cylinder, the piston rod of which is directly mounted onto the plunger of the syringe (Fig. 4). The pneumatic system is controlled by a time-switch. It operates a magnet valve which controls the supply of compressed air. The sequence of a rain cycle is a follows: After a signal from the time-switch the magnet valve is opened for a few seconds, compressed air flows to the cylinders, its piston rod moves to the left so that the syringe is emptied and the liquid pressed to the rain-head. The magnet valve is closed and a spring construction in the syringe and cylinder then re-sets the system to its original position. The syringe is refilled with influent solution during re-setting. Two one-way valves control the direction of the liquid stream. They should be of very good quality. If compressed air is not available, a cylinder of compressed air can be used because only minute amounts are needed per cycle. The pneumatic system (cylinder, syringe and magnet valve) is mounted on a framework in a way that cylinder and syringe are exactly in line.

\section{The filter}

Before a soil column is placed in the rack, the hollow auger-head is unscrewed and replaced by a filter which is also screwed onto the bottom of the column. It is filled 
with sand. The lower plate is perforated with holes of $2 \mathrm{~mm}$ diameter and covered by a sheet of nylon. A special construction of the filter is required to prevent the development of air bubbles in the boundary layer between soil core and filter. Good drainage is then blocked. Experience showed that two things are important: the sand in the filter should be arranged in a special way and a close contact should exist between soil core and filter. These two requirements are fulfilled in the following way. The sand is put in the filter in three layers in fractions of decreasing diameter, from bottom to top. The fractions are $2.0-0.6 \mathrm{~mm}, 0.6-0.3 \mathrm{~mm}$ and $0.3-0.16 \mathrm{~mm}$, respectively. The sand is slightly moistened by a spray-gun. It is frozen at $-25{ }^{\circ} \mathrm{C}$ overnight to facilitate easy handling and then screwed onto the bottom of the column until it comes into close contact with the soil core.

\section{Facilities of the installation}

The soil column installation is provided with some facilities in order to be able to change experimental conditions if required. The soil water potential can be varied and experiments can be done under aerobic or anaerobic conditions.

\section{Soil water potential}

At the position where effluent drops from the column the water potential equals zero. This position represents the phreatic level. So all positions upwards in the column are at under-pressure. This means that the wider capillaries in the soil are not filled with water and do not contribute to water migration. Even the space be-

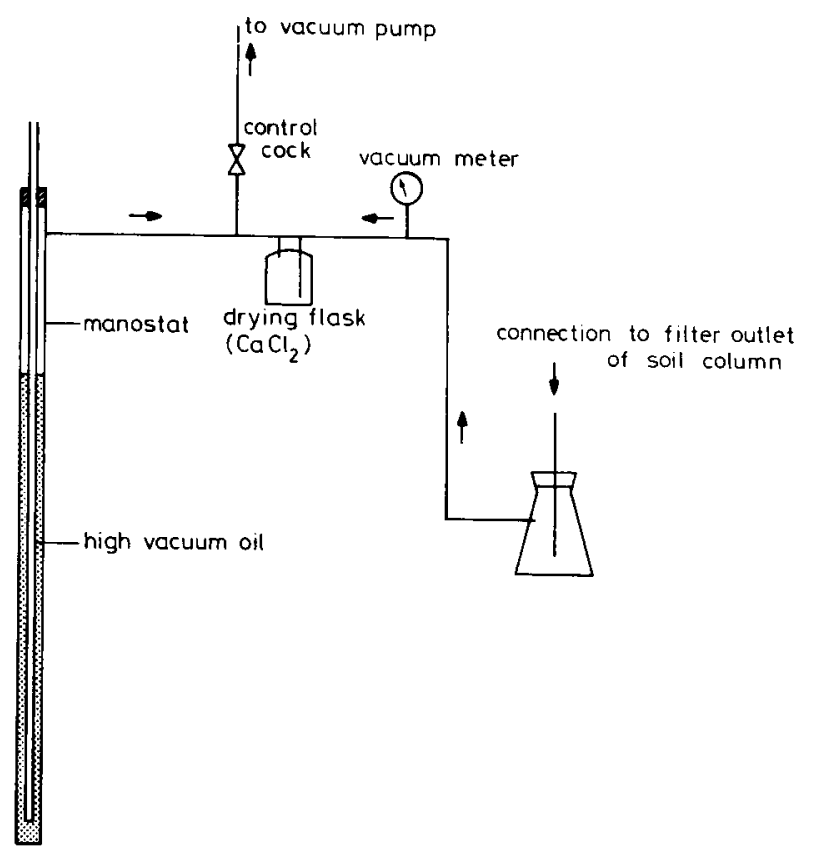

Fig. 5. Scheme of the underpressure system. 
tween soil core and column wall can be considered as such a wide capillary and will therefore not be filled with water when migration experiments are started. It is therefore unnecessary to take soil cores of exactly the same diameter as the diameter of the perspex cylinder. This facilitates the preparation of the soil columns (see section 'Preparation of the soil columns').

In order to simulate lower phreatic level additional under-pressure is applied as shown in Fig. 5. As migration experiments usually take rather a long time - from several months to more than one year - precautions have been taken to avoid the water vapour entering the vacuum line. A drying flask filled with $\mathrm{CaCl}_{2}$ is placed between effluent container and vacuum pump and the manostat is filled with high vacuum oil. The under-pressure is calculated from the length of the oil column times the oil density. Tensiometer gauges are inserted in the soil columns to measure the water potential. Holes are easily bored in the cylinder wall and are also easily sealed with home-made perspex-chloroform cement.

\section{Anaerobic conditions}

Experiments sometimes require anaerobic conditions. The columns are then kept in a nitrogen atmosphere (see Fig. 3), while the last traces of oxygen are removed by micro-organisms. To speed up the microbiological action nutrients may be added; a mixture of $\mathrm{NH}_{4} \mathrm{NO}_{3}$ and glucose with a $\mathrm{C} / \mathrm{N}$ ratio of 20 is very useful. If very low redox potentials are required, a mixture of $\mathrm{NH}_{4} \mathrm{Cl}$ and glucose may also be useful.

To check the removal of oxygen a small siphon flask is connected to the soil column and filled with a reversible indicator solution (see Fig. 3). The nitrogen gas leaves the column through this siphon flask. The indicator solution is a methylene blue solution, based on Fildes' method described by McClung \& Lindberg (1957). The oxidized form is blue, the reduced form is colourless. Our modification is prepared as follows. Three stock solutions are made, consisting of:

a) $6.0 \mathrm{ml}$ of $0.1 \mathrm{~N} \mathrm{NaOH}$ made up to $100 \mathrm{ml}$ with distilled water;

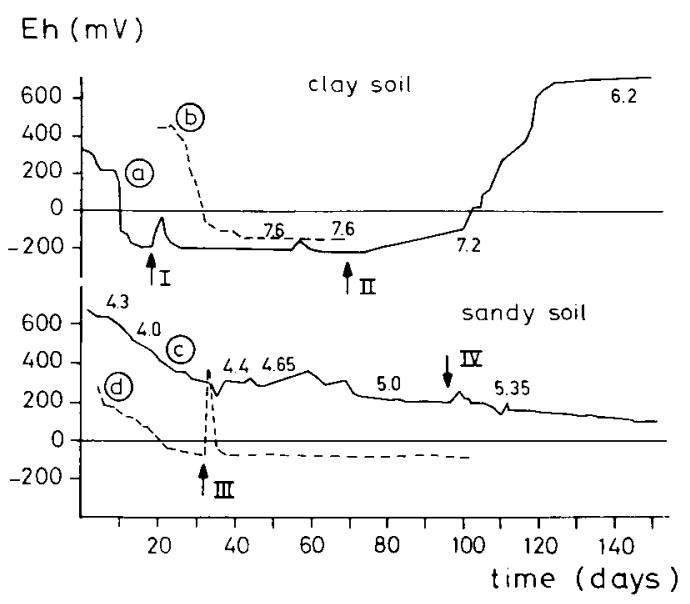

Fig. 6. Redox potentials in soil columns. Curves $a$ and $b$ : redox potentials measured in the same clay column, a at a depth of $17 \mathrm{~cm}, \mathrm{~b}$ at a depth of $40 \mathrm{~cm}$. Curve c: sandy soil, potential measured at a depth of $10 \mathrm{~cm}$. Curve d: identical sandy soil, but inoculated with sewage sludge, Arabic figures: $\mathrm{pH}$ values. Time: number of days after the replacement of the normal air by nitrogen gas. Roman figures: I, III and IV: column opened for inspection; II: nitrogen gas replaced by normal air. 
b) $3.0 \mathrm{ml}$ of $0.5 \%$ aqueous methylene blue made up to $100 \mathrm{ml}$ with distilled water;

c) $6.0 \mathrm{~g}$ glucose in $100 \mathrm{ml}$ of distilled water with a small crystal of thymol.

When an indicator is needed equal parts of each solution are mixed and the mixed solution heated until the blue colour disappears.

The redox potential is measured by inserting electrodes into the soil column in the same way as described for the soil water potential measurements. Boring a hole in the column wall and soil core may cause a rather long-lasting disturbance of the redox potential, especially in sandy soils. In clay soils the redox potential is buffered rather well, as shown in Fig. 6 . The figure also shows the progress of the redox potential with time for different soils at different depths.

\section{Measurements}

Migration patterns of the investigated compound using the installation described can be obtained in three ways:

- measurements can be made in the effluent to check the course of concentration with time;

- when the compound can be labelled with a $\gamma$-emitting nuclide a soil column scanner can be used;

- the soil core can be cut into slices and the concentration measured in each slice.

A soil column scanner has been constructed which automatically scans the soil column and records the activity per layer on punch tape. During this scanning the column rotates to eliminate local irregularities, so that a good average over the column is obtained.

The moisture content in the column can also be measured by a 'double-beam' scanner. The absorption of both weak and hard gamma-radiation beams are determined. Because the absorption depends on the thickness and density of the layer, it is possible to calculate the moisture content. Such a double-beam scanner is described by Stroosnijder \& de Swart (1974).

Furthermore it is of course possible to measure all variables which can be determined via electrodes (to be inserted through the column wall). For the study of saline soils the conductivity must also be mentioned.

An instrument which presses the soil core from the perspex cylinder in adjustable steps was also constructed. The soil core it then cut in layers to the desired thickness.

\section{Acknowledgments}

The authors wish to acknowledge the assistance received from K. E. Wit (Institute for Land and Water Management Research, ICW) in the early days of their soil column preparations.

\section{References}

McClung, L. S. \& R. B. Lindberg, 1957. The study of obligately anaerobic bacteria. Manual of micro-biological methods. McGraw-Hill, New York/London, p. 120-122.

Stroosnijder, L. \& J. G. de Swart, 1974. Column scanning with simultaneous use of ${ }^{241} \mathrm{Am}$ and ${ }^{137}$ Cs gamma radiation. Soil Sci. 118: 61-69. 